\title{
CAPTAÇÃO DE ÁGUA POR SISTEMA FOTOVOLTAICO PARA CONSUMO HUMANO NA LOCALIDADE RURAL ILHA CAIAPÓS, SÃO ROMÃO, MINAS GERAIS
}

\section{Water abstraction by photovoltaic system for human consumption in rural Caiapos island location, Sao Romao, Minas Gerais}

\author{
Samuel Alves Boitrago', Saulo Soares Santos ${ }^{2}$, Adão Helivan Ribeiro Gonçalves ${ }^{3}$, \\ Marco Antônio Ramos Canela ${ }^{4}$
}

Recebido em 30 de agosto de 2015; recebido para revisão em 03 de março de 2016; aceito em 23 de abril de 2015; disponível on-line em 04 de julho de 2016.

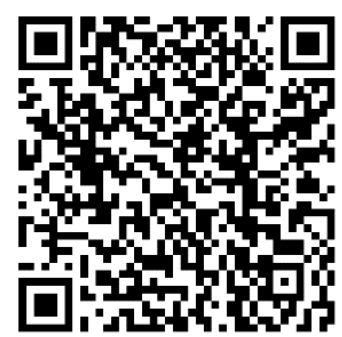

PALAVRAS CHAVE:

Sistema de recalque fotovoltaico;

Captação de água;

Comunidade rural;

Energia solar.

\section{KEYWORDS:}

Photovoltaic settlement system;

Water catchment;

Rural community;

Solar energy.

* Contato com os autores:

1 e-mail: samuelboitrago@hotmail.com (S. A. Boitrago)

Graduando em Engenharia Civil pelas Faculdades Integradas do Norte de Minas.

2e-mail: sauloeng.civil@yahoo.com.br (S. S. Santos)

Graduando em Engenharia Civil pelas Faculdades Integradas do Norte de Minas.

${ }^{3}$ e-mail: a_helivan@hotmail.com (A. H. R. Gonçalves)

Graduando em Engenharia Civil pelas Faculdades Integradas do Norte de Minas.

${ }^{4}$ e-mail: marcoantonio.canela@funorte.edu.br (M. A. R. Canela)

Docente do ICESP - Promove de Brasília. 


\section{INTRODUÇÃO}

O aproveitamento das águas subterrâneas para o abastecimento público pode oferecer soluções simples e de grande viabilidade técnica e econômica principalmente no abastecimento de pequenas comunidades e núcleos populacionais de zona rural, pois a captação de água subterrânea subsuperficiais, por poços rasos, drenos e as aflorantes são de fácil implantação, operação, manutenção e baixo custo de construção (HELLER e PÁDUA, 2010).

Devido à água subterrânea ocorrer sob uma camada de material filtrante não saturado ou relativamente pouco permeável, este manancial encontra-se, comparativamente, melhor protegido dos agentes poluidores que afetam rapidamente a qualidade da água dos rios (REBOUÇAS, 2001).

Para Heller e Pádua (2010) as instalações para o abastecimento de água, que se insere no contexto mais amplo de saneamento, deve fornecer água com qualidade, regularidade e de forma acessível para a população.

Conforme Fraidenraich e Vilela (1999), as bombas solares são preferencialmente utilizadas para atender demanda de pequenas vazões de água, tal como as requeridas por pequenos povoados de 100 a 1.000 habitantes, e para o atendimento de necessidades agrícolas moderadas.

Dentre as inúmeras tecnologias de bombeamento de água existentes, a opção fotovoltaica se mostra uma das mais promissoras para o abastecimento de populações sem acesso à rede elétrica convencional e localizada em zonas remotas. A tecnologia fotovoltaica apresenta vantagens em vários aspectos, iniciando pelo fato de o recurso solar ocorrer em todo o globo terrestre, sendo sua utilização uma questão solucionável mediante dimensionamento. Além disso, evita gastos permanentes na aquisição e transporte de combustível, bem como a emissão de gases poluentes e de ruído na geração. Trata-se de uma tecnologia consolidada tecnicamente, de alta confiabilidade e com uma vida útil do gerador de mais de 25 anos (NOGUEIRA et al., 2013).

Para Fraidenraich e Vilela (1999) apesar de ainda pouco difundidos, os sistemas de bombeamento fotovoltaico apresentam muitas vantagens. O fato de ser um equipamento modular permite acompanhar o crescimento da demanda de água do local com o aumento da potência do equipamento. $O$ gerador fotovoltaico não possui partes móveis, o que facilita sua instalação e manutenção. Outra vantagem do sistema de bombeamento solar é o "bom casamento" entre o perfil temporal do recurso energético e a demanda de água, tanto do ponto de vista diário como sazonal, já que os períodos de elevada insolação coincidem com os de maior consumo.

A atual preocupação com a preservação do meio ambiente e a possível escassez na oferta de combustíveis fósseis, nos induz a busca de tecnologias para aperfeiçoar a utilização de fontes alternativas de energia, menos poluentes e que minimizam impactos ambientais. A energia solar é abundante, renovável e não polui, sendo a solução ideal para abastecer eletricamente áreas remotas desprovidas do fornecimento de energia elétrica. Devido suas características é relevante para o sistema ambiental, pois o Sol tem potencial energético extremamente elevado e incomparável a qualquer outro sistema de energia, sendo a fonte básica e indispensável para praticamente todas as fontes energéticas utilizadas pelo homem (GOMES, 2012).

A radiação solar consiste em toda radiação eletromagnética que incide sobre a superfície terrestre proveniente do Sol (QUERINO et al. 2006). Essa radiação pode ser captada e transformada em alguma forma de energia utilizável pelo homem, seja diretamente como fonte de energia térmica, podendo também ser convertida em energia elétrica, por meio de um sistema fotovoltaico (APRILE et al. 2006).

A irradiação solar depende de fatores como localidade, estação do ano, composição atmosférica, cobertura de nuvens e a forma da superfície. O dimensionamento e o desempenho de uma central fotovoltaica dependem de alguns parâmetros: radiação global, radiação direta, radiação difusa, horas de sol, temperatura média e 
variável, é necessário um levantamento histórico dos seus valores para garantir um melhor aproveitamento do sistema de geração ao longo do ano (WANDERLEY e CAMPOS, 2013).

De acordo com a ANEEL (2008) o Brasil é um dos países que apresenta uma grande intensidade de radiação solar em toda a sua extensão, o que torna bastante importante utilizarmos esse potencial como fonte alternativa para geração de energia elétrica.

Conforme Gastaldi, Souza e Mesquita (2004) os maiores índices de radiação solar são observados na Região Nordeste, com evidência no Vale do São Francisco, onde a média anual é de aproximadamente $6 \mathrm{kWh} / \mathrm{m}^{2}$.dia. No entanto, a Região Nordeste é pobre em recursos hídricos para a geração de energia elétrica.

\section{OBJETIVOS}

Verificar a viabilidade técnica e econômica de implantação de um sistema de bombeamento utilizando a energia solar fotovoltaica para captação de água na localidade de Ilha Caiapós, São Romão, Minas Gerais.

\section{METODOLOGIA}

Para o dimensionamento do sistema de energia solar fotovoltaica para captação de água, levou-se em consideração a vazão necessária para atendimento da população, o número de placas solares e a característica da bomba submersa.

O estudo foi realizado no Município de São Romão, localizado na Região Norte do estado de Minas Gerais, à distância de 511 km de Belo Horizonte. Seu território está inserido na bacia do Rio São Francisco, sendo sua posição geográfica determinada pelo paralelo 1622'07" de latitude Sul em sua interseção com o meridiano 4504'10" de longitude oeste.

A comunidade denominada Ilha Caiapós localiza-se no Rio São Francisco, ao lado da cidade de São Romão. O acesso à comunidade é difícil, necessitando de barco para atravessar o Rio São Francisco.

\subsection{DIMENSIONAMENTO DA POPULAÇÃO E VAZÕES}

De acordo com o IBGE (2010), a população residente na localidade e de 67 habitantes. Dessa forma visando atender um horizonte de projeto de vinte anos projetou-se um crescimento populacional pelo método geométrico, adotado um coeficiente fixo de crescimento com base na população inicial do ano de 2010.

A taxa de crescimento é dada por meio das Equações de 1 a 4:

$$
\frac{\mathrm{dP}}{\mathrm{dt}}=\mathrm{K}_{\mathrm{g}} \cdot \mathrm{P}
$$

A equação da projeção é dada por:

$$
\mathrm{P}_{\mathrm{t}}=P_{o} \times(1+i)^{\left(t-t_{o}\right)}
$$

Coeficientes:

$$
\begin{aligned}
& \mathrm{K}_{\mathrm{g}}=\frac{\ln \mathrm{P}_{2}-\ln \mathrm{P}_{0}}{\mathrm{t}_{2}-\mathrm{t}_{0}} \\
& \mathrm{i}=\mathrm{e}^{\mathrm{k}_{\mathrm{g}}}-1
\end{aligned}
$$

\section{Sendo:}

dP/dt: Taxa de crescimento da população em função do tempo;

Pt: População estimada no ano t (hab.);

$P_{0}, P_{1}, P_{2}$ : Populações nos anos $t_{0}, t_{1}, t_{2}$;

$\mathrm{k}_{\mathrm{g}}$, i: Coeficientes (a obtenção dos coeficientes pela análise da regressão é preferível, já que se pode utilizar toda a série de dados existentes e não apenas $P_{0}, P_{1}$ e $P_{2}$ ).

Com base nas Equações de 1 a 4 e com os dados retirados do IBGE para a população do ano de 2010, foi possível gerar a Tabela 1 com a projeção populacional da comunidade para o ano de 2014.

Os parâmetros utilizados para dimensionamento das vazões do sistema de abastecimento de água foram. 
- Regime de abastecimento 24 h/dia;

- Regime de produção máximo $6 \mathrm{~h} /$ dia;

- Consumo "per capita" q = 140 L/habxdia;

- Coeficiente do dia de maior consumo $\mathrm{K}_{1}=1,2$;

- Coeficiente da hora de maior consumo $\mathrm{K}_{2}=1,5$;

- Horizonte de Projeto 20 anos;

- Índice de atendimento $100 \%$.

Segundo Heller e Pádua (2010) para povoado rural com população abaixo de 5.000 habitantes o consumo varia entre 90 a 140 L/habxdia.

Para a determinação das vazões de projeto utilizou-se as Equações 5, 6 e 7:

$$
\begin{gathered}
Q_{d . \text { med }}=\frac{P \times q_{p c}}{86400} \\
Q_{D M C}=Q_{d . \text { med }} \times K_{1} \\
Q_{H M C}=Q_{d . \text { med }} \times K_{1} \times K_{2}
\end{gathered}
$$

Sendo:

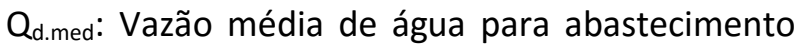
$(\mathrm{L} / \mathrm{s})$;

QDMc: Vazão média do dia de maior consumo (L/s);

Qнмс: Vazão média da hora de maior consumo (L/s);

P: População no ano de interesse de abastecimento (hab);

qpc: Consumo "per capita" de consumo de água de abastecimento (l/hab d).

A partir dos parâmetros apresentados foi possível calcular as vazões média, máxima diária, máxima horária e de produção necessárias para o dimensionamento do sistema de captação de água. Para a quantidade de horas de funcionamento levou-se em consideração o tempo de isolação na localidade bem como o volume de reservação dimensionado para dias de pouca irradiação solar. Os cálculos da projeção populacional com horizonte de projeto e cálculo de vazões estão apresentados na Tabela 2.

\section{TABELA 1: Dados e informações populacional.}

\begin{tabular}{|c|c|c|c|c|c|c|c|c|c|c|c|}
\hline \multirow[b]{2}{*}{ Ano } & \multirow[b]{2}{*}{ Civil } & \multirow{2}{*}{$\begin{array}{l}\text { População } \\
\text { (hab) }\end{array}$} & \multirow{2}{*}{$\begin{array}{c}\text { População } \\
\text { atendida } \\
\text { (hab) }\end{array}$} & \multirow{2}{*}{$\begin{array}{c}\text { Índice de } \\
\text { perdas } \\
(\%)\end{array}$} & \multicolumn{4}{|c|}{ Vazões consumidas $(\mathrm{L} / \mathrm{s})$} & \multicolumn{2}{|c|}{$\begin{array}{l}\mathrm{N}^{\mathrm{o}} \text { de horas de } \\
\text { funcion. (h/dia) }\end{array}$} & \multirow{2}{*}{$\begin{array}{c}\text { Reservação } \\
\text { necessária } \\
\left(\mathbf{m}^{\mathbf{3}}\right)\end{array}$} \\
\hline & & & & & Med. & $\begin{array}{c}\text { Max. } \\
\text { dia. }\end{array}$ & $\begin{array}{l}\text { Max. } \\
\text { hor. }\end{array}$ & Prod. & $\begin{array}{c}\text { Q } \\
\text { Med. }\end{array}$ & $\begin{array}{l}\text { Q Max. } \\
\text { dia. }\end{array}$ & \\
\hline PROJ & 2015 & 74 & 74 & 25 & 0,12 & 0,14 & 0,22 & 0,58 & 3,70 & 4,45 & 7,78 \\
\hline 1 & 2016 & 75 & 75 & 25 & 0,12 & 0,15 & 0,22 & 0,58 & 3,76 & 4,51 & 7,90 \\
\hline 2 & 2017 & 76 & 76 & 25 & 0,12 & 0,15 & 0,22 & 0,59 & 3,82 & 4,58 & 8,02 \\
\hline 3 & 2018 & 77 & 77 & 25 & 0,13 & 0,15 & 0,23 & 0,60 & 3,87 & 4,65 & 8,14 \\
\hline 4 & 2019 & 79 & 79 & 25 & 0,13 & 0,15 & 0,23 & 0,61 & 3,93 & 4,72 & 8,26 \\
\hline 5 & 2020 & 80 & 80 & 25 & 0,13 & 0,16 & 0,23 & 0,62 & 3,99 & 4,79 & 8,38 \\
\hline 6 & 2021 & 81 & 81 & 25 & 0,13 & 0,16 & 0,24 & 0,63 & 4,05 & 4,86 & 8,51 \\
\hline 7 & 2022 & 82 & 82 & 25 & 0,13 & 0,16 & 0,24 & 0,64 & 4,11 & 4,93 & 8,63 \\
\hline
\end{tabular}

\begin{tabular}{ccccc} 
Ano & Hab. / resid. & Resid. & Coeficiente i & População \\
2014 & 4,56 & 16 & 0,015 & 73 \\
\hline & \multicolumn{4}{c}{ FONTE: Acervo do autor (2015). }
\end{tabular}

TABELA 2: Projeção populacional e cálculo das vazões. (Continua). 
TABELA 2: Projeção populacional e cálculo das vazões. (Continuação).

\begin{tabular}{|c|c|c|c|c|c|c|c|c|c|c|c|}
\hline \multirow{2}{*}{ Ano } & \multirow{2}{*}{ Civil } & \multirow{2}{*}{$\begin{array}{c}\text { População } \\
\text { (hab) }\end{array}$} & \multirow{2}{*}{$\begin{array}{c}\text { População } \\
\text { atendida } \\
\text { (hab) }\end{array}$} & \multirow{2}{*}{$\begin{array}{c}\text { Índice de } \\
\text { perdas } \\
(\%)\end{array}$} & \multicolumn{4}{|c|}{ Vazões consumidas (L/s) } & \multicolumn{2}{|c|}{$\begin{array}{l}N^{0} \text { de horas de } \\
\text { funcion. (h/dia) }\end{array}$} & \multirow{2}{*}{$\begin{array}{c}\text { Reservação } \\
\text { necessária } \\
\left(\mathbf{m}^{3}\right)\end{array}$} \\
\hline & & & & & Med. & $\begin{array}{c}\text { Max. } \\
\text { dia. }\end{array}$ & $\begin{array}{l}\text { Max. } \\
\text { hor. }\end{array}$ & Prod. & $\begin{array}{c}\mathbf{Q} \\
\text { Med. }\end{array}$ & $\begin{array}{l}\text { Q Max. } \\
\text { dia. }\end{array}$ & \\
\hline 8 & 2023 & 83 & 83 & 25 & 0,14 & 0,16 & 0,24 & 0,65 & 4,17 & 5,01 & 8,76 \\
\hline 9 & 2024 & 85 & 85 & 25 & 0,14 & 0,16 & 0,25 & 0,66 & 4,24 & 5,08 & 8,90 \\
\hline 10 & 2025 & 86 & 86 & 25 & 0,14 & 0,17 & 0,25 & 0,67 & 4,30 & 5,16 & 9,03 \\
\hline 11 & 2026 & 88 & 88 & 25 & 0,14 & 0,17 & 0,26 & 0,68 & 4,40 & 5,28 & 9,24 \\
\hline 12 & 2027 & 89 & 89 & 25 & 0,14 & 0,17 & 0,26 & 0,69 & 4,45 & 5,34 & 9,35 \\
\hline 13 & 2028 & 90 & 90 & 25 & 0,15 & 0,18 & 0,26 & 0,70 & 4,50 & 5,40 & 9,45 \\
\hline 14 & 2029 & 92 & 92 & 25 & 0,15 & 0,18 & 0,27 & 0,72 & 4,60 & 5,52 & 9,66 \\
\hline 15 & 2030 & 93 & 93 & 25 & 0,15 & 0,18 & 0,27 & 0,72 & 4,65 & 5,58 & 9,77 \\
\hline 16 & 2031 & 95 & 95 & 25 & 0,15 & 0,18 & 0,28 & 0,74 & 4,75 & 5,70 & 9,98 \\
\hline 17 & 2032 & 96 & 96 & 25 & 0,16 & 0,19 & 0,28 & 0,75 & 4,80 & 5,76 & 10,08 \\
\hline 18 & 2033 & 97 & 97 & 25 & 0,16 & 0,19 & 0,28 & 0,75 & 4,85 & 5,82 & 10,19 \\
\hline 19 & 2034 & 99 & 99 & 25 & 0,16 & 0,19 & 0,29 & 0,77 & 4,95 & 5,94 & 10,40 \\
\hline 20 & 2035 & 100 & 100 & 25 & 0,16 & 0,19 & 0,29 & 0,78 & 5,00 & 6,00 & 10,50 \\
\hline
\end{tabular}

Foram considerados os dados de 20 anos, garantindo assim o atendimento a população futura e funcionamento adequado dos equipamentos.

\subsubsection{Dimensionamento e escolha da bomba}

A partir da vazão de produção e da profundidade estimada que o poço opere com nível dinâmico de 30 metros e estático de 15 metros de profundidade. Considerando as perdas de cargas localizadas e por trechos gerou-se a curva do sistema com a bomba para a escolha do conjunto motobomba.

Assim a posição onde $\mathrm{o}$ ponto de operação, obtidos pelas interseções da curva de vazão da bomba versus as curvas de altura manométrica do sistema estão determinados na Tabela 3.

Por meio dos dados levantados, adotouse o conjunto moto bomba com as características mostradas na Tabela 4.

A bomba escolhida apresenta como desvantagens o motor de indução, na utilização em sistemas fotovoltaicos, pois a baixa tolerância às variações na tensão de alimentação quando em plena carga, incompatibilidade com as tensões produzidas pelo painel fotovoltaico e as correntes de partida deste tipo de máquina podem chegar a várias vezes o valor da corrente nominal, o que exigir, portanto um sobre dimensionamento dos painéis fotovoltaicos.

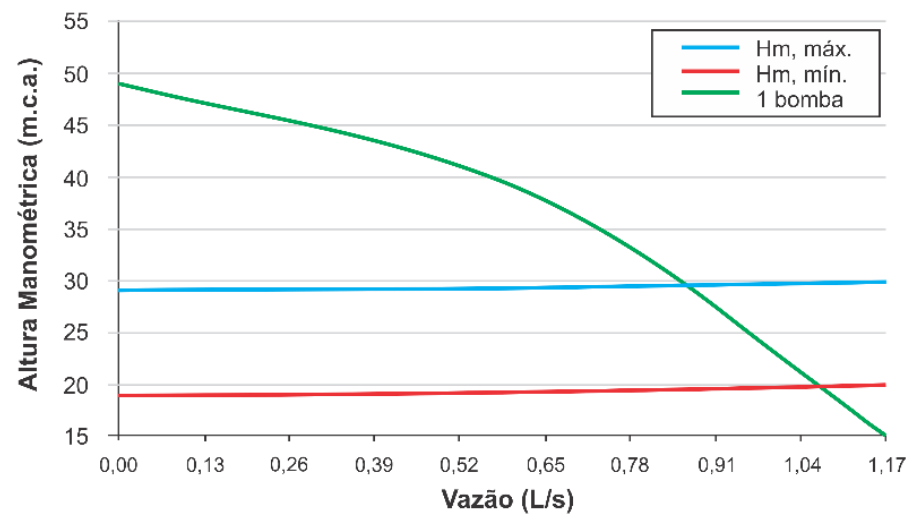

FIGURA 1: Curva da bomba x sistema. Fonte: Acervo do autor (2015). 


\begin{tabular}{|ccc|}
\hline \multicolumn{2}{|c|}{ TABELA 3: Ponto de operação. } \\
\hline Parâmetro & $\mathrm{H}_{\mathrm{m}, \text { mín }}$ & $\mathrm{H}_{\mathrm{m} \text {,máx }}$ \\
\hline $\mathrm{Q}=$ vazão $(\mathrm{L} / \mathrm{s})$ & 1,11 & 0,85 \\
$\mathrm{H}_{\mathrm{m}}=$ altura manométrica $(\mathrm{m})$ & 19,74 & 29,45 \\
\hline & \multicolumn{2}{c}{ FONTE: Acervo do autor (2015). }
\end{tabular}

\begin{tabular}{|c|c|c|}
\hline Modelo de referência & 4R3 IB & \\
\hline Tipo & Submersa & \\
\hline \multicolumn{3}{|l|}{ Carcaça do Motor } \\
\hline Número de bombas & 1 & \\
\hline Potência nominal & 0,50 & $\mathrm{cV}$ \\
\hline Vazão & 0,78 & $1 / \mathrm{s}$ \\
\hline Altura manométrica & 30,00 & $\mathrm{~m}$ \\
\hline Rotação & 3.450 & $\mathrm{rpm}$ \\
\hline
\end{tabular}

Entretanto com o avanço e a redução de custos na eletrônica de potência, essas desvantagens é contornadas com o uso de conversores CC-CA.

\subsubsection{Tipos de sistemas fotovoltaicos}

Os sistemas fotovoltaicos são divididos basicamente em duas categorias: isolados ou autônomos e os conectados à rede. A escolha de qual sistema utilizar depende da necessidade e da disponibilidade de redes públicas no local.

\subsubsection{Dimensionamento do sistema fotovoltaico}

A Energia Solar Fotovoltaica é a energia que provém da conversão direta da luz em eletricidade (Efeito Fotovoltaico) (APRILE et al. 2006). O efeito fotovoltaico decorre de uma diferença de potencial nos extremos de uma estrutura de material semicondutor, produzida pela absorção da luz. A célula solar ou fotovoltaica é a unidade fundamental do processo de conversão de energia solar em energia elétrica. No entanto a eficiência dessa conversão depende da radiação solar que incide sobre a superfície dessas células convertendo-as em energia elétrica (CERPCH, 2011).
O norte de Minas Gerais apresenta características climáticas bastante promissoras para este tipo de captação de energia, essa comprovação está presente nos Atlas Solarimétrico de Minas Gerais. Estudos realizado pela CEMIG e o governo do Estado aponta índices de radiação solar suficientes em mais da metade da área do Estado com geração de energia solar de grande porte, seja termoelétrica ou fotovoltaica, chegando a uma radiação solar direta anual de $2.700 \mathrm{kwh} / \mathrm{m}^{2}$ no verão e de cerca de $2.200-2.400 \mathrm{kwh} / \mathrm{m}^{2}$ em bases anuais.

A região tem radiação solar anual média 6,5 a $7,0 \mathrm{kWh} / \mathrm{m}^{2} /$ dia, (Figura 3).

Como a localidade de estudo não é contemplada com energia elétrica convencional, utilizou-se o sistema fotovoltaico autônomo, também chamado de sistema isolado, são utilizados em lugares onde não há redes elétricas convencionais como: embarcações, residências em zonas rurais que não há o fornecimento de energia, em ilhas, dentre outros lugares que não são atendidos pela rede pública e se faz necessário o uso da energia elétrica (VILLALVA; GAZOLI, 2012).

Grande parte dos sistemas fotovoltaicos autônomos opera na tensão de $12 \mathrm{~V}$. Inversores, 


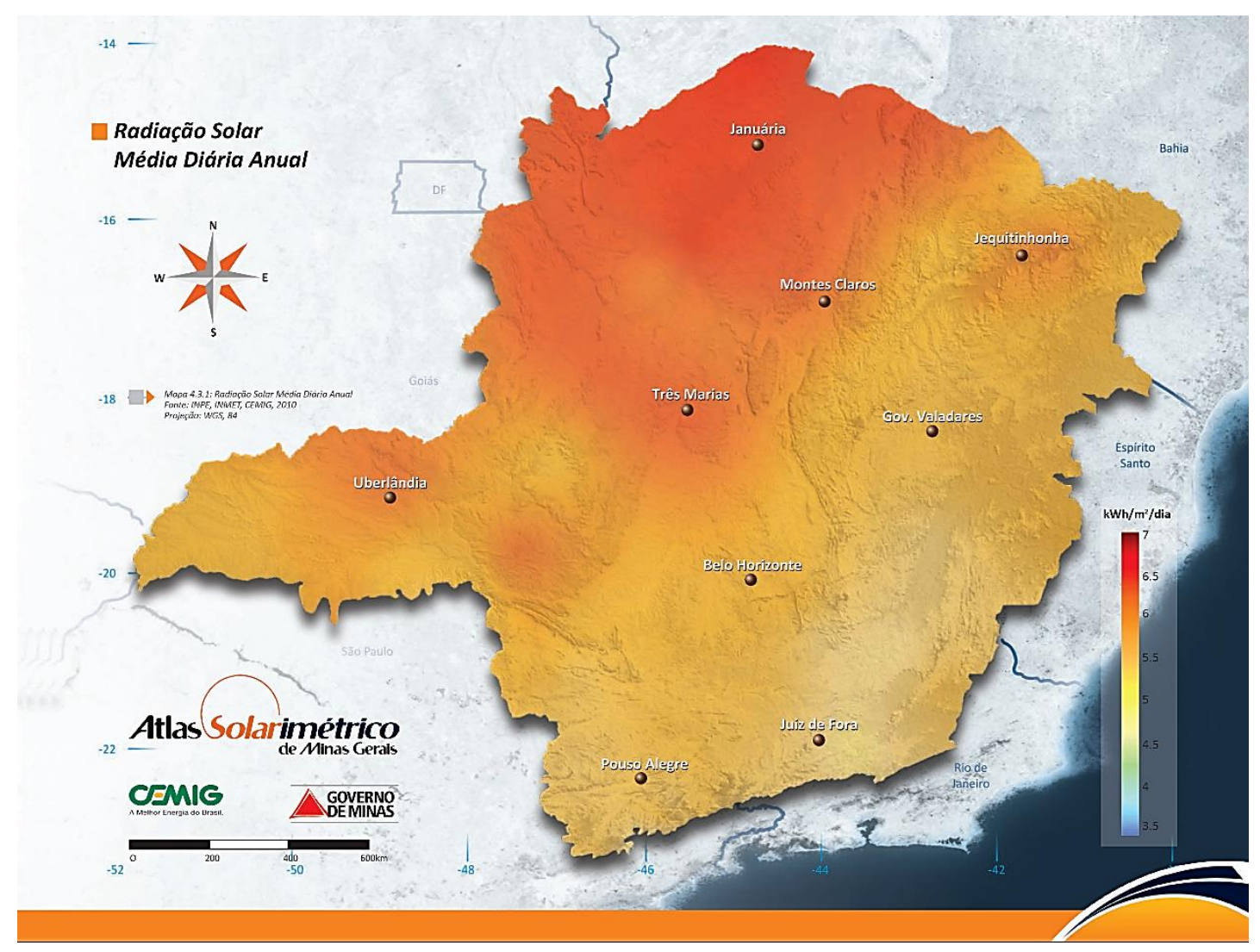

FIGURA 1: Mapa da Radiação Solar Média Diária Anual.

FONTE: Atlas Solarimétrico - CEMIG (2015).

baterias e controladores de carga para 12 V são os mais encontrados no mercado. Existem módulos fabricados especialmente para serem compatíveis com esses sistemas autônomos de $24 \mathrm{~V}$.

No dimensionamento do sistema não foi contemplado bateria para reserva de energia, pois tem custos elevados e o sistema previa um funcionamento de 6 horas diárias, adotando um reservatório para atender a demanda quando o sistema fotovoltaico não estivesse em funcionamento ou em dias pouco ensolarado, onde a produção de energia fosse reduzida.

O cálculo de energia necessária para alimentar a bomba é feito pela relação entre a potência dos aparelhos e pelo número de horas que ele é utilizado.

A energia gasta pelo sistema em um dia nublado foi calculada pela Equação 8:

$$
E C=P \times T
$$

Sendo:

$\mathrm{EC}=$ Energia consumida em watts-horas [Wh];

$\mathrm{P}=$ Potência em watts $[\mathrm{W}]$;
$\mathrm{T}=$ Tempo de uso em horas [h];

$\mathrm{EC}=720 \mathrm{Wh} \times 2$ dias $=1.440 \mathrm{Wh}$.

Para definir a quantidade de módulos a ser usado no sistema, calculou-se a produção do módulo escolhido. Com as características em STC (Condições padrão de teste no modulo) ou NOCT (Condições normais de operação do modulo).

O cálculo da energia produzida pelo módulo em NOCT foi feito pela Equação 9:

$$
P M=I S C \times V
$$

Sendo:

PM = Potência do Modulo [W];

Isc = Corrente de curto-circuito do módulo $[\mathrm{A}]$;

$\mathrm{V}_{\text {máx. }}=$ Tensão Máxima $[\mathrm{V}]$;

$P M=8,11 \mathrm{~A} \times 27,20 \mathrm{~V}=220,59 \mathrm{~W}$.

Onde o cálculo da energia produzida pelo modulo nesse método foi realizado pela Equação 10: 


\section{Sendo:}

$\mathrm{EP}=$ Energia produzida pelo modulo diariamente

[Wh];

PM = Potência do Modulo [W];

HS = Horas diárias de insolação $[h]$;

$E P=220,59 \mathrm{~W} \times 6 \mathrm{~h}=1.323,54 \mathrm{Wh} /$ dia.

Assim, a Tabela 5 apresenta a demanda necessária para o funcionamento da bomba.

O número total de módulos necessários no sistema foi calculado por meio da Equação 11:

$$
\mathrm{N}=\mathrm{EC} / \mathrm{EP} \quad \text { Eq. [11] }
$$

Sendo:

$\mathrm{N}=$ Número de módulos;

$\mathrm{EC}=$ Energia consumida em watts-horas [Wh];

$\mathrm{EP}=$ Energia produzida pelo modulo diariamente [Wh].

Onde:

$\mathrm{N}=2.536 \mathrm{Wh} / 1.323,54 \mathrm{Wh}=1,94$;

Dessa forma, utilizou-se dois módulos de modelo (Yingli Solar 245 W).

\subsubsection{Inversor de corrente}

O inversor é um dispositivo eletrônico que transforma a eletricidade de tensão e corrente contínuas (CC) em tensão e corrente alternadas (CA) (CARVALHO, 2013). Foi considerado para o experimento um inversor de corrente de $24 \mathrm{~V}$ para $110 \mathrm{~V}$.

\subsubsection{Reservação}

Por não ter adotado bateria no dimensionamento, o sistema somente bombeou água enquanto houve irradiação solar suficiente, para justificar a vazão, todo excedente de energia elétrica do sistema, foi armazenado em forma de energia potencial gravitacional, armazenando-se a água excedente em um reservatório elevado. Assim, a fim de garantir o abastecimento de água continuo foi dimensionado um reservatório com capacidade de $15 \mathrm{~m}^{3}$, cujo volume também se justifica para o atendimento durante o período noturno, ou períodos de pouca insolação.

\subsection{INVESTIMENTO INICIAL}

Para a implantação do sistema foi levantado os custos relativos à implantação do sistema de captação de água por sistema fotovoltaico, as cotações foram retiradas do comercio local e cotações online sem considerar o BDI (Benefícios e Despesas Indiretas), na Tabela 8 está apresentada à estimativa de custos para o investimento inicial.

\begin{tabular}{cccccc}
\hline \multicolumn{7}{c}{ TABELA 5: Característica da bomba. } \\
\hline $\begin{array}{c}\text { Quantidade de } \\
\text { bombas }\end{array}$ & Tensão $[\mathrm{V}]$ & $\begin{array}{c}\text { Potência da } \\
\text { bomba }[\mathrm{CV}]\end{array}$ & $\begin{array}{c}\text { Potência total do } \\
\text { sistema }[\mathrm{W}]\end{array}$ & $\begin{array}{c}\text { Operação } \\
\text { Horas/dia }\end{array}$ & $\begin{array}{c}\text { Energia } \\
\text { consumida } \\
{[\text { Wh/dia] }}\end{array}$ \\
1 & 127 & 0,5 & 422,63 & 6 & 2.536 \\
\hline
\end{tabular}

TABELA 6: Investimento inicial.

\begin{tabular}{lcc}
\multicolumn{1}{c}{ Descrição } & Número de Unidades & Custos \\
\hline Módulo fotovoltaico Yingli Solar 245W & 2 & $\mathrm{R} \$ 2.360,00$ \\
Inversor Wagan Tech Pro Line 2007-430 A - & 1 & $\mathrm{R} \$ 1.985,00$ \\
24V/110V & 1 & $\mathrm{R} \$ 1.609,00$ \\
Bomba Leão modelo 4R3 & 2 & $\mathrm{R} \$ 2.538,00$ \\
Suporte para Painel Fotovoltaicos de até & 1 & $\mathrm{R} \$ 566,90$ \\
250Wp & 1 & $\mathrm{R} \$ 11.980,00$ \\
Cabo Duflex10mm Rolo com 100m & 9 & $\mathrm{R} \$ 369,00$ \\
Reservatório 15m 3 & 8 & $\mathrm{R} \$ 34,80$ \\
Tubo PVC PBA DN 50mm 6m & 1 & $\mathrm{R} \$ 3.000,00$ \\
Conexões & 1 & $\mathrm{R} \$ 2.500,00$ \\
Perfuração de poço até 30 metros & 26 & $\mathrm{R} \$ 26.942,70$ \\
Despesas com mão de Obra & & \\
Total & & \\
\hline
\end{tabular}




\section{RESULTADOS}

O custo total para a implantação pode ser considerado elevado, inicialmente, contudo levando em consideração a vida útil do sistema por um período de vinte anos, dividido pelos meses e pela população, é um custo relativamente baixo. Não foi realizado tratamento na água, pois o mesmo é efetivado no sistema operacional. Vale ressaltar que na captação subterrânea há possibilidades do tratamento ser simplificado com custos reduzidos.

O sistema em NOCT apresentou vazão de $0,78 \mathrm{~L} / \mathrm{s}$ em um funcionamento de 6 horas diárias, totalizando um volume de $16,85 \mathrm{~m}^{3}$ de água. Esse volume atende as necessidades da população da Ilha Caiapós para o final de plano, cujo volume é de $10,50 \mathrm{~m}^{3}$ diários.

A Figura 3 apresenta um desenho esquemático do sistema fotovoltaico.

\section{CONCLUSÃO}

O sistema de captação de água por energia solar fotovoltaica apresentou-se econômico e viável, tecnicamente, para implantação em pequenas comunidades rurais. Os custos para a implantação não são elevados, além de ser uma benfeitoria de uso coletivo para atendimento de toda comunidade, utilizando uma fonte de energia renovável e com o conceito de energia limpa. A geração da energia na própria comunidade, através da tecnologia fotovoltaica, é de suma importância, visto que poupa altos investimentos para extensão de energia elétrica convencional.

A implantação do sistema de bombeamento na comunidade não resolve todos os problemas de saneamento, visto que, além da captação deve ser realizado o tratamento, mesmo que simplificado, e a distribuição, ressaltando a participação ativa da população na compreensão e manutenção dessa tecnologia.

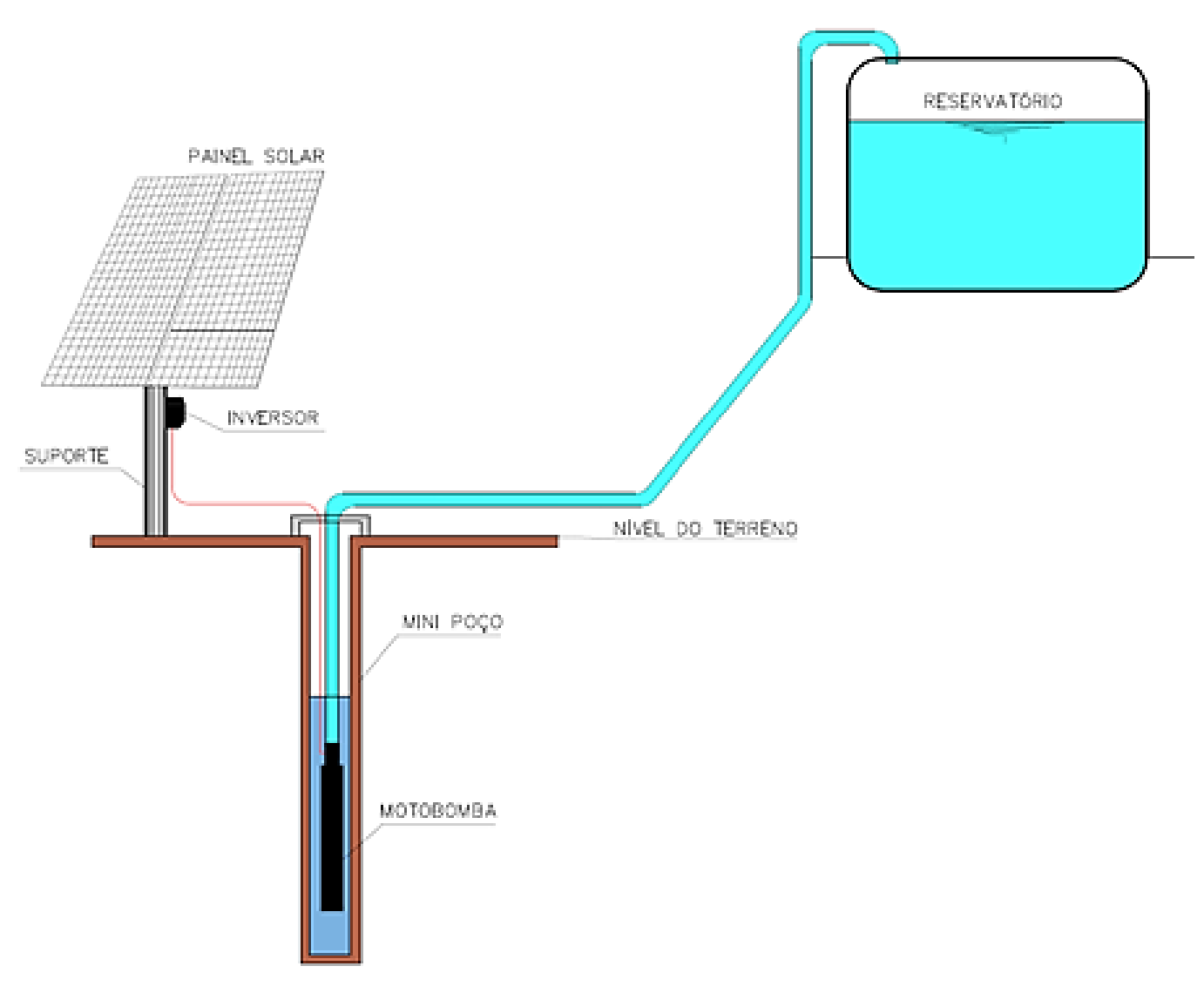

FIGURA 2: Desenho esquemático do sistema fotovoltaico.

FONTE: Acervo do autor (2015). 


\section{REFERÊNCIAS}

ANEEL. Agencia Nacional de Energia Elétrica. Altas de Energia Elétrica do Brasil: Parte 2 Fontes Renováveis. Brasília 2008.

ATLAS SOLARIMETRICO DE MINAS GERAIS. Seção: Faça o download do ATLAS aqui! Disponível em: <http://www.atlassolarimetricomg.com.br/>. Acesso em: 25 fev. 2015.

APRILE, C.; PINTO, G.; DUARTE, J. G. C.; MATOS, M. H. S.; CORINTI, R. Transmissão de calor: Conversão de energia solar em energia térmica e elétrica. UNESP, p. 1-4, 2006.

CERPCH. Centro Nacional de Referência em Pequenas Centrais Hidrelétricas. FONTES RENOVAVEIS: ENERGIA SOLAR. Disponível em: <http://www.cerpch.unifei.edu.br/solar.php>. Acesso em 23 fev. 2015.

CARVALHO, C. R. F. Sistema fotovoltaico isolado: uma aplicação pratica no projeto xapuri. 2013. 46f. Trabalho de conclusão de Curso (Licenciatura Formas Alternativas de Energia) - UNIVERSIDADE FEDERAL DE LAVRAS, 2013. FRAIDENRAICH, N.; VILELA, O. C. Avanços em sistemas de abastecimento de água com bombeamento fotovoltaico para comunidades rurais. Revista Brasileira de Recursos Hídricos, v. 4, n. 3, p. 69-81, 1999.

GASTALDI, A. F.; SOUZA, T. M.; MESQUITA, R. P. Geração de energia elétrica com célula solar fotovoltaica para população rural de baixa renda. Centro de Energias Renováveis - UNESP - Guaratinguetá. 2004.

GOMES, C. P. Energia Solar: Utilização como fonte de energia alternativa. Bolsista de Valor: Revista de divulgação do Projeto Universidade Petrobras e IF Fluminense, v. 2, n. 1, p. 159-163, 2012.

HELLER, L.; PÁDUA, V. L. Abastecimento de água para consumo humano. 2 ed. Belo Horizonte: Editora UFMG, 2010. 418 p.

IBGE - Instituto Brasileiro de Geografia e Estatística. Censo 2010. Disponível em:<http://www.ibge.gov.br>. Acesso em: 23 fev. 2015.

NOGUEIRA, C.; NOGUEIRA, H. M.; GARCIA, F. T.; LOPES, L. F. Utilização de sistemas solar e eólico no bombeamento de água para uso na irrigação. Entrevista, v. 15, n. 2, p. 127-135, 2013.
QUERINO, C.A.S.; MOURA, M.A.L.; R.F.F. LYRA, MARIANO, G.L. Avaliação e comparação de radiação solar global e albedo com ângulo zenital na região Amazônica. Rev. Bras. de Meteorologia. v. 21(3a), p. 42 - 49, 2006.

REBOUÇAS, A. C. Água e desenvolvimento rural. Estudos Avançados, v. 15, n. 43, p. 327-344, 2001.

VILLALVA, M. G.; GAZOLI, J. R. Energia solar fotovoltaica conceitos e aplicações: Sistemas Isolados e Conectados à Rede. 1 ed.; São Paulo: Érica, 2012. 224 p.

WANDERLEY, A. C. F.; CAMPOS, A. L. P. S. Perspectivas de inserção da energia solar fotovoltaica na geração de energia elétrica no Rio Grande do Norte. HOLOS, v. 3, n. 29, p. 3-14, 2013. 